\title{
Deformation twinning and residual stress in calcite studied with synchrotron polychromatic X-ray microdiffraction
}

\author{
Kai Chen • Martin Kunz • Nobumichi Tamura • \\ Hans-Rudolf Wenk
}

Received: 15 October 2010/Accepted: 19 February 2011/Published online: 20 March 2011

(C) The Author(s) 2011. This article is published with open access at Springerlink.com

\begin{abstract}
Microstructures of deformed calcite in marble from the Bergell Alps are studied by using a microfocused polychromatic synchrotron X-ray beam. The high spatial resolution, together with orientation and strain resolutions, reveals twin plane orientation, multiple twin lamellae, and strain distributions associated with the twins. Single and multiple mechanical twins on $e=\{01 \overline{1} 8\}$ systems are confirmed. Residual stresses are derived from the strain tensor that is derived from Laue diffraction patterns. Average lattice strains from several hundred to over one thousand microstrains are detected in a deformed marble from the Bergell Alps. Such strains suggest 60-120 MPa residual stresses. A detailed study of strain components shows that shear stresses on twin planes are completely released.
\end{abstract}

Keywords Calcite - Twinning - Residual stress - Laue microdiffraction

\section{Introduction}

Mechanical twinning was first discovered in the rhombohedral mineral calcite $\mathrm{CaCO}_{3}$ (Pfaff 1859) and is an important mechanism for plastic deformation at high shear stresses in this mineral (Burkhard 1993). Twins occurring on the lattice plane $e=\{01 \overline{1} 8\}$ are easily introduced into

K. Chen · H.-R. Wenk ( $₫)$

Department of Earth and Planetary Science,

University of California, Berkeley, CA 94720, USA

e-mail: wenk@berkeley.edu

M. Kunz · N. Tamura

Advanced Light Source, Lawrence Berkeley Laboratory,

1 Cyclotron Road, Berkeley, CA 94720, USA calcite by applying a knife blade across the edge of the cleavage rhomb. Such twins form, in general, at low temperature and high stress and cause discontinuous shearing on the grain scale, which in turn gives rise to considerable strain incompatibilities at grain boundaries. Calcite twins are common in marble from many tectonic environments and are easily visible as lamellar structures. Because of their widespread occurrence, calcite twins have potential to be used as paleopiezometers to record stresses during mountain building (e.g., Rowe and Rutter 1990; Lacombe and Laurent 1992, 1996; Lacombe et al. 1992, 2009; Ferrill 1998; González-Casado et al. 2006; Amrouch et al. 2010). In view of this, several groups worked to quantify the strain/stress relationships associated with the twin structure over the last 40 years (Spang 1972, 1974; Chinn and Konig 1973; Spang and Van Der Lee 1975; Jamison and Spang 1976; Laurent et al. 1981; Evans and Groshong 1994). The microstructure of twins has been studied extensively by using transmission electron microscopy (TEM), which led to the observation of high dislocation densities along twin boundaries, suggesting local stress concentrations (e.g., Barber and Wenk 1979; De Bresser 1996; Larsson and Christy 2008).

Diffraction is considered the standard method for quantitative measurements of residual lattice strain/stress (Noyan and Cohen 1987). However, the main difficulty for studying the local lattice strain associated with deformation twinning in calcite is the requirement of high spatial resolution. With recent development of synchrotron X-ray microdiffraction techniques, it becomes possible to evaluate the crystal orientation as well as strain with a micron- to submicron-scale spatial resolution on a sample surface. A microfocused high-brilliance polychromatic X-ray beam can be produced with Kirkpatrick-Baez (KB) mirrors at synchrotron facilities (Liu et al. 2005), and Laue diffraction 
patterns (LPs) can be recorded by large-area two-dimensional (2D) CCD detectors. The spatial resolution of this technique is only limited by the X-ray beam size, the scanning step size, and the sample thickness. Local orientation, strain/stress distribution, and plastic deformation information are obtained by analyzing the diffraction pattern and comparing experimentally measured peak positions and peak shapes with those from an unstrained lattice (e.g., Tamura et al. 2003).

\section{Experimental}

In this paper, we report the microstructure of calcite deformation twins in a coarse-grained metamorphic marble. The sample Brg 861 from Pass del Cam in the Bergell Alps, Switzerland, is a recrystallized Triassic limestone of greenschist metamorphic grade. It is moderately deformed with large grains, which range from hundreds of microns to a couple of millimeters in diameter and contain lamellar twins. An uncovered standard petrographic thin section (approximately $50 \mu \mathrm{m}$ thick) was used as sample for the diffraction experiments. We determined the lattice strain tensors at micron spatial resolution over two areas of the thin section. One area is $140 \times 140 \mu \mathrm{m}$ in size containing a grain with crossing twins, and a second one, $250 \times 100 \mu \mathrm{m}$ in size, contains a grain with a set of secondary twins that terminate at the primary twin boundaries. Based on stiffness and strain tensors, stress tensors were also determined and mapped to reveal the stress distributions in the host and twin domains, especially those close to the twin boundaries.

Synchrotron Laue X-ray microdiffraction was performed on Beamline 12.3.2 at the Advanced Light Source (ALS) of the Lawrence Berkeley National Laboratory (LBNL). An X-ray beam with a wide energy spectrum ( $5 \mathrm{keV}<\mathrm{E}<22 \mathrm{keV}$ ) was focused with two sets of focusing mirrors to a $1 \times 1 \mu \mathrm{m}$ size (Kunz et al. 2009a). The thin-sectioned marble sample, mounted on a highresolution sample stage, tilted by $45^{\circ}$ with respect to the incoming X-ray beam and positioned at the X-ray focal point with the assistance of a laser triangulation system to within $5 \mu \mathrm{m}$, was raster-scanned through the microfocused $\mathrm{X}$-ray beam. An LP was recorded in reflection geometry at each scanning position with a 133-mm-diameter MAR133 $\mathrm{X}$-ray CCD detector, which was positioned at about $8 \mathrm{~cm}$ above the sample surface and its normal oriented $90^{\circ}$ with respect to the incident X-ray beam. The diffraction geometry, including the sample-to-detector distance, the detector tilt angles, and the central channel position of the detector, was calibrated with an unstrained single crystal of Si mounted next to the marble sample. A set of fixed righthanded sample coordinates, of which the $\mathbf{z}$-axis was defined normal to the sample surface and the $\mathbf{x}$-axis within the sample surface and perpendicular to X-ray beam direction, was established for crystal orientation representation. The experimental configuration and the LPs collected were similar to those described elsewhere for quartz (Kunz et al. 2009b).

\section{Data analysis}

All LPs were automatically indexed with rhombohedral lattice parameters $(a=b=c=6.375 \AA, \alpha=\beta=\gamma=$ $46.07592^{\circ}$, Graf 1961), using the software package XMAS (Tamura et al. 2009). After indexing, based on the unstrained lattice parameters, strain refinement was performed by a least-squares approach to match the position of calculated peaks with the measured positions. Five parameters $(a / c, b / c, \alpha, \beta$, and $\gamma)$ were refined. The absolute lattice parameters cannot be determined with the Laue technique, which relies on polychromatic radiation, since CCD detectors cannot differentiate energies of detected $\mathrm{X}$-ray photons. After initial analysis with a rhombohedral unit cell, all orientation matrices were converted into hexagonal setting by applying the following transformation matrix for comparison with existing literature:

$$
\left[\begin{array}{c}
a_{1} \\
a_{2} \\
a_{3} \\
c
\end{array}\right]_{\mathrm{H}}=\left[\begin{array}{ccc}
1 & -1 & 0 \\
0 & 1 & -1 \\
-1 & 0 & 1 \\
1 & 1 & 1
\end{array}\right]\left[\begin{array}{l}
a \\
b \\
c
\end{array}\right]_{\mathrm{R}}
$$

where $\mathbf{a}_{1}, \mathbf{a}_{2}, \mathbf{a}_{3}, \mathbf{a}, \mathbf{b}, \mathbf{c}$ are vectors representing the orientation of the lattice parameters, and $\left[\begin{array}{l}a_{1} \\ a_{2} \\ a_{3} \\ c\end{array}\right]_{\mathrm{H}}$ and $\left[\begin{array}{l}a \\ b \\ c\end{array}\right]_{\mathrm{R}}$ are the orientation matrices in hexagonal and rhombohedral settings, respectively. In this paper, all lattice coordinates and Miller indices $\{h k i l\}$ refer to the hexagonal setting unless otherwise stated.

The crystal lattice is distorted from its unstrained symmetry under stress because of the anisotropy of stress and stiffness tensors; thus, the orientation matrix was refined with and without symmetry constraint. The difference between these two results then allows the determination of the deviatoric strain tensor of the crystal region that is investigated. Since no lattice volume information is provided with the Laue diffraction technique, the strain tensors described in this paper are all deviatoric strains. The deviatoric strain tensor, $\boldsymbol{\varepsilon}_{\mathrm{ij}}{ }^{\prime}$, is defined as follows:

$\varepsilon_{\mathbf{i j}}^{\prime}=\varepsilon_{\mathrm{ij}}-\varepsilon_{\mathrm{M}} \delta_{\mathrm{ij}}$

where $\varepsilon_{\mathbf{i j}}$ and $\boldsymbol{\delta}_{\mathbf{i j}}$ are the full strain tensor and the unit tensor, respectively. $\varepsilon_{\mathrm{M}}=\frac{\varepsilon_{11}+\varepsilon_{22}+\varepsilon_{33}}{3}$, and the term $\varepsilon_{\mathrm{M}} \boldsymbol{\delta}_{\mathbf{i j}}$ is a measurement of dilatation or volume change. The 
orientation and strain resolution depends mainly on the calibration of the diffraction geometry and the number and shape of diffraction peaks. They are estimated to be approximately $0.01^{\circ}$ and $10^{-4}$, respectively, for this study.

Stress was calculated by applying Hooke's law $\boldsymbol{\sigma}_{\mathbf{i}}=\mathbf{C}_{\mathbf{i j}} \boldsymbol{\varepsilon}_{\mathbf{j}}$, where $\boldsymbol{\sigma}_{\mathbf{i}}, \mathbf{C}_{\mathbf{i j}}$, and $\boldsymbol{\varepsilon}_{\mathbf{j}}$ are the stress tensor (2nd rank), stiffness tensor (4th rank), and strain tensor (2nd rank), respectively. In our study, the stiffness tensor reported by Chen et al. (2001) was employed. However, it is worthwhile to note that $\mathbf{C}_{i j}$ is anisotropic so that the stress tensor obtained in this way is not necessarily the deviatoric stress, i.e., the summation of the diagonal components of stress tensor is not zero for non-cubic crystals.

As an indication of the magnitude of deviatoric strain and stress, von Mises equivalent strain and von Mises equivalent stress are defined as follows (Liu 2005):

$\varepsilon_{e q}=\frac{2}{3} \sqrt{\frac{\left(\varepsilon_{11}-\varepsilon_{22}\right)^{2}+\left(\varepsilon_{22}-\varepsilon_{33}\right)^{2}+\left(\varepsilon_{33}-\varepsilon_{11}\right)^{2}+6\left(\varepsilon_{12}^{2}+\varepsilon_{13}^{2}+\varepsilon_{23}^{2}\right)}{2}}$

$\sigma_{e q}=\sqrt{\frac{\left(\sigma_{11}-\sigma_{22}\right)^{2}+\left(\sigma_{22}-\sigma_{33}\right)^{2}+\left(\sigma_{33}-\sigma_{11}\right)^{2}+6\left(\sigma_{12}^{2}+\sigma_{13}^{2}+\sigma_{23}^{2}\right)}{2}}$

where $\varepsilon_{\mathrm{ij}}$ are the deviatoric strain components as defined above.

\section{Results}

\section{Crossing twins}

In one of the two grains that were studied, crossing twins are observed with the petrographic microscope, as shown in Fig. 1a. In this study, a $140 \times 140 \mu \mathrm{m}$ area, indicated by the square, is scanned with a $2-\mu \mathrm{m}$ step size. We assume the big uniform domain is the host domain and small lamellae are twin lamellae. Strong diffraction peaks of a host domain are detected in all 4,900 LPs at approximately constant peak positions, indicating that the orientation of this domain is almost constant within the measured area. In some regions, diffraction peaks from twin domains are also observed. The host domain is first indexed in all the LPs (as indicated by the squares in Fig. 1b), and the orientation map is shown in Fig. 1c. Subsequently, the diffraction peaks from the host domain are subtracted from each LP by the software, and LPs of twin domains which produced relatively weak peaks (indicated by circles in Fig. 1b) were indexed again, and the map is displayed in Fig. 1d. From Fig. 1c, it can be seen that the c-axis of the host domain lies in a narrow range $\left(\sim 1^{\circ}\right)$ around about $26^{\circ}$ off the $\mathbf{x}$-axis. White spots in the orientation map indicate that the LP failed to be indexed. Figure 1d, which maps the angles between $\mathbf{c}$-axis and $\mathbf{x}$-axis, shows several twin lamellae in
Fig. 1 a Optical micrograph of a calcite grain containing crossing twins (crossed polarizers). b A typical Laue diffraction pattern showing strong diffraction peaks from host domain (some marked with squares) and relatively weak diffraction peaks from twin domain (some marked with circles). Orientation maps showing the angles between sample $\mathbf{x}$-axis and crystal $\mathbf{c}$-axis of $\mathbf{c}$ the host domain and $\mathbf{d}$ the twin domains 1, 2, and 3 as indicated by the labeled arrows
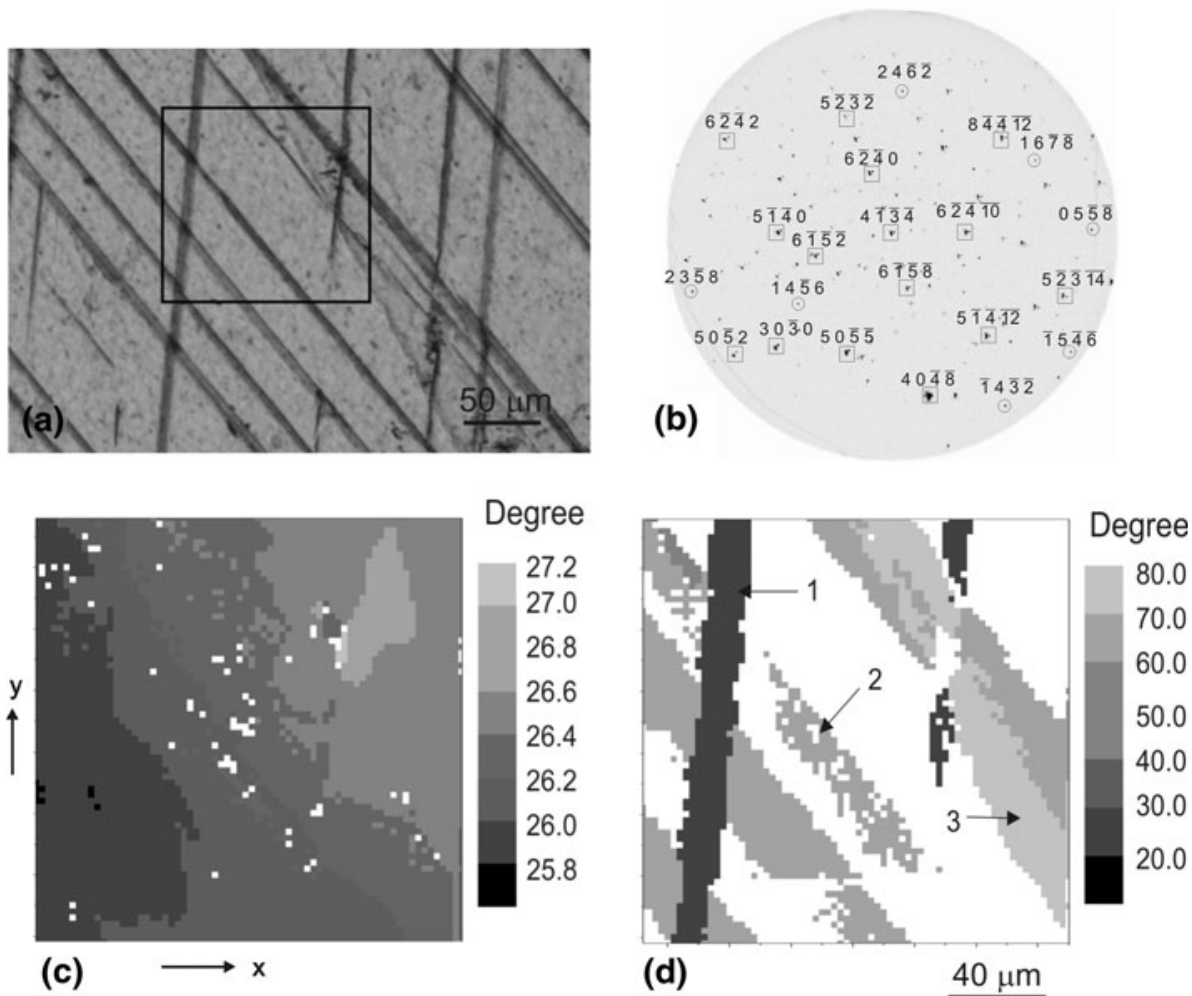
the selected region, with three distinct orientations as suggested by different gray shades. The traces of the twin lamellae with similar orientation are approximately parallel to one another and are indicated by the parallel bands with same shades in the orientation maps. The three twin orientations were identified as \#1, \#2, and \#3.

Stereographic projections are used to display the orientation relationship of the twin domains and the host domain. The poles of $\{01 \overline{1} 8\}$ planes of the host domain are plotted in Fig. 2. These are found to correspond, respectively, to the poles of the $(0 \overline{1} 1 \overline{8}),(1 \overline{1} 08)$, and $(10 \overline{1} \overline{8})$ planes of the twin Domains 1, 2, and 3 defined in Fig. 1c. The poles to the twin boundaries of Domain 1 and 2 are both close to the sample surface (i.e., the twin boundaries are nearly normal to the sample surface), while the twin boundaries of Domain 3 are oblique to the surface. As a cross-check, we also calculated the rotation angles and axes between each twin domain and the host domain. It is found that the rotation angles from the host domain to each twin domain are about $52.6^{\circ}-53.0^{\circ}$ along $\left.<11 \overline{2} 0\right\rangle$ directions, which is very close to the published value $\left(52.5^{\circ}\right)$ for $e$-twinning (e.g., Mügge 1883).

Figures 3 and 4 display strain distributions in host domain and twin domains, respectively, in laboratory coordinates. The laboratory coordinate system is a Cartesian coordinate system, the $\mathbf{z}$-axis of which is defined to be normal to the sample surface, and the $\mathbf{x}$-axis perpendicular to the incident X-ray beam. In regions away from the twin lamellae, such as the area to the top-right corner of the scan, the strain magnitude is small; in the region close to the twin lamellae, it is higher $\left(\sim>1.5 \times 10^{-3}\right)$. The twin boundaries can be visualized from the strain maps of the

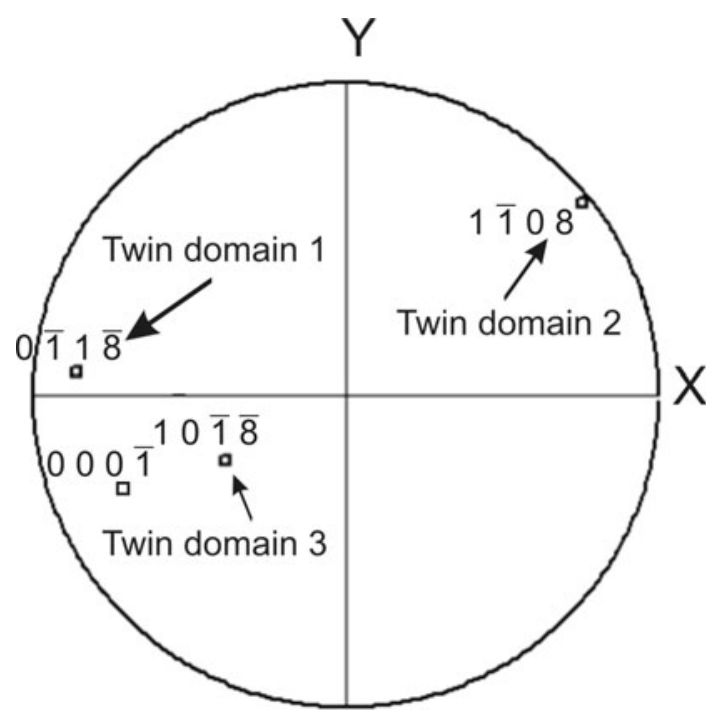

Fig. 2 Stereographic projection showing the c-axis (0001) and $\{1 \overline{1} 08\}$ poles of the host domain. Each member of the $\{1 \overline{1} 08\}$ zone of the host corresponds to a $\{1 \overline{1} 08\}$ pole of one of the three twin domains host domain, as indicated by the lines on the $\varepsilon_{\mathrm{yy}}$ plot in Fig. 3. In this reference system, high off-diagonal strain components are observed in the host domain. From Fig. 4, it is suggested that the normal strain in the $\mathbf{x}$-direction $\left(\varepsilon_{\mathrm{xx}}\right)$ is tensile (yellow, red), while the strain along the $\mathbf{y}$-axis is compressive (blue), regardless of the crystal orientations of the twin domains.

\section{Stopping twins}

In the second region we selected to study, thin secondary twin lamellae are observed as well as thick primary twin lamellae (Fig. 5a). However, since the primary twin domains have similar thickness as the host domains, it is difficult to distinguish between primary "twin" and "host" domains. We will analyze this in the discussion section. The primary twin boundaries are oblique to the sample surface, while boundaries of the secondary twins are nearly perpendicular to the sample surface. Secondary twins terminate at primary twin boundaries (stopping twins). A $250 \times 100 \mu \mathrm{m}$ area was scanned with a $2 \mu \mathrm{m}$ step size. We denote the three domains as Domain 1, Domain 2, and the secondary twin Domain 3. Figure 5b-d shows the 2D orientation maps of Domains 1, 2, and 3, respectively. Since the primary twin boundaries are oblique and synchrotron hard X-rays are penetrative in calcite, Domain 1 and Domain 2 overlap in these 2D maps. Domain 1 has its c-axis about $11^{\circ}$ oblique to the $\mathbf{x}+\mathbf{y}$ direction (Fig. 5b), but the c-axis of Domain 2 is $37^{\circ}$ off the sample surface normal (z-axis) (Fig. 5c). Domain 3 is difficult to map because diffraction peaks are very weak compared to those of Domain 1. Therefore, only three secondary twin lamellae, the orientations of which are shown in Fig. 5d, were detected within the Domain 1 region. Domain 3 has the a-axis aligned along the $\mathbf{z}$-axis.

As in the first region we studied, we plot the poles of (1108) and (0118) planes of Domain 1. It is observed that (1108) poles of Domain 1 and 2 are in the same orientation, as are the $(01 \overline{1} 8)$ poles of Domain 1 and Domain 3 . Calculations indicate that Domain 1 is rotated by $51.6^{\circ}$ and $52.5^{\circ}$ around $\langle 11 \overline{2} 0\rangle$ axes to form Domain 2 and Domain 3 , respectively, consistent with $e$-twins.

From the pole figure (Fig. 6a), we can also see that the normal of the twin plane between Domain 1 and 2 is about $45^{\circ}, 63^{\circ}$, and $56^{\circ}$ oblique with respect to the $\mathbf{x}-, \mathbf{y}$-, and $\mathbf{z}$-axis of the sample coordinates, respectively. Based on this geometry, the thin-section thickness and twin lamellae thickness can be estimated. First, we draw schematically the cross section of the sample perpendicular to the twin plane (Fig. 6b), where $c f$ indicates the width of twin lamellae, $b c$ the lamellae gap, $c e$ the twin plane, and $d e$ the sample thickness. Measured from the orientation map, ad and $b c$ are approximately 84 and $21 \mu \mathrm{m}$, respectively, and 

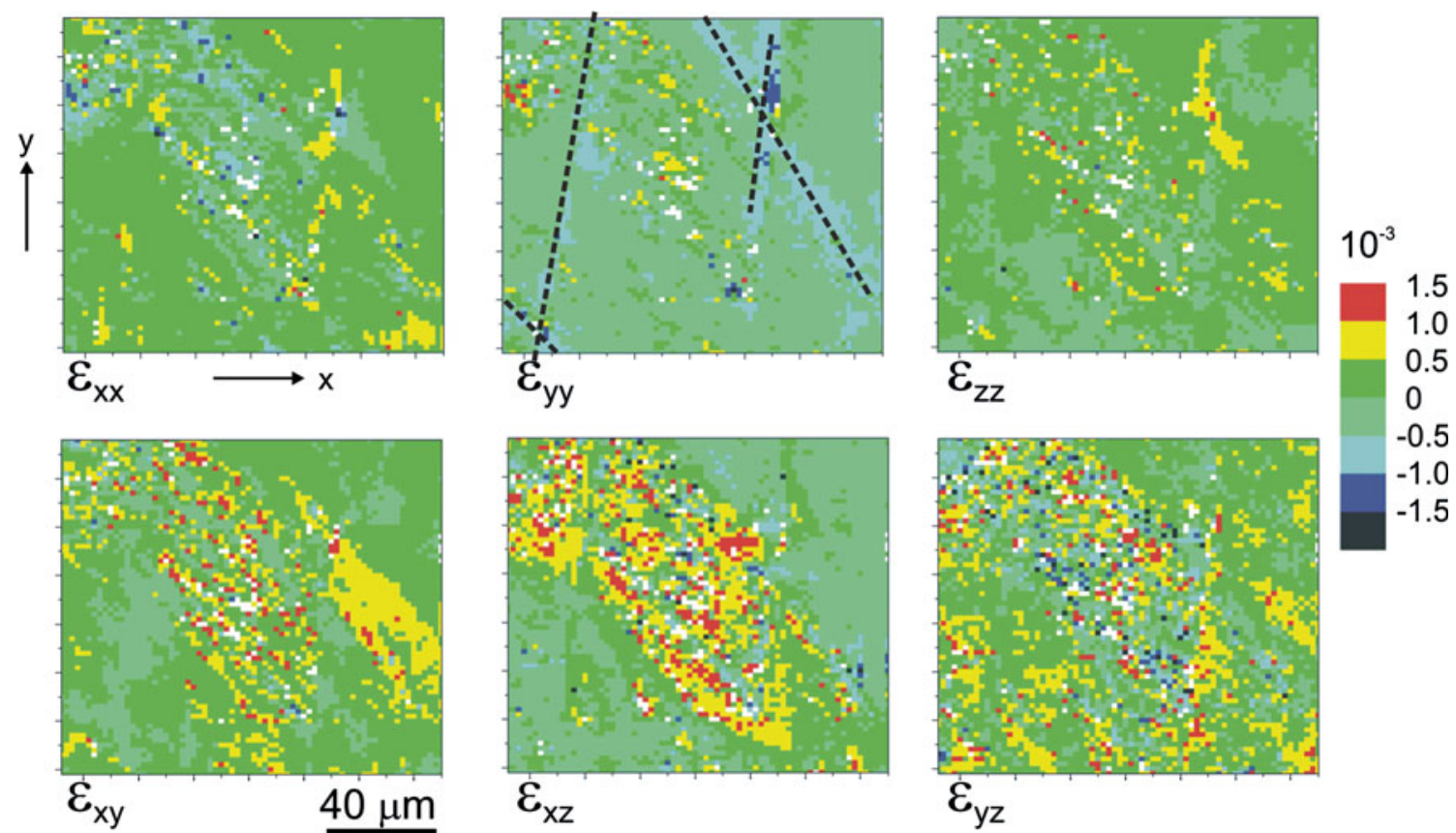

Fig. 3 Maps of strain components $\varepsilon_{x x}, \varepsilon_{x y}, \varepsilon_{x z}, \varepsilon_{y y}, \varepsilon_{y z}, \varepsilon_{z z}$ in the host domain. The coordinate system refers to laboratory coordinates $\mathbf{x}, \mathbf{y}$, and $\mathbf{z}$. Units are microstrain, positive values indicate extension, negative values compression
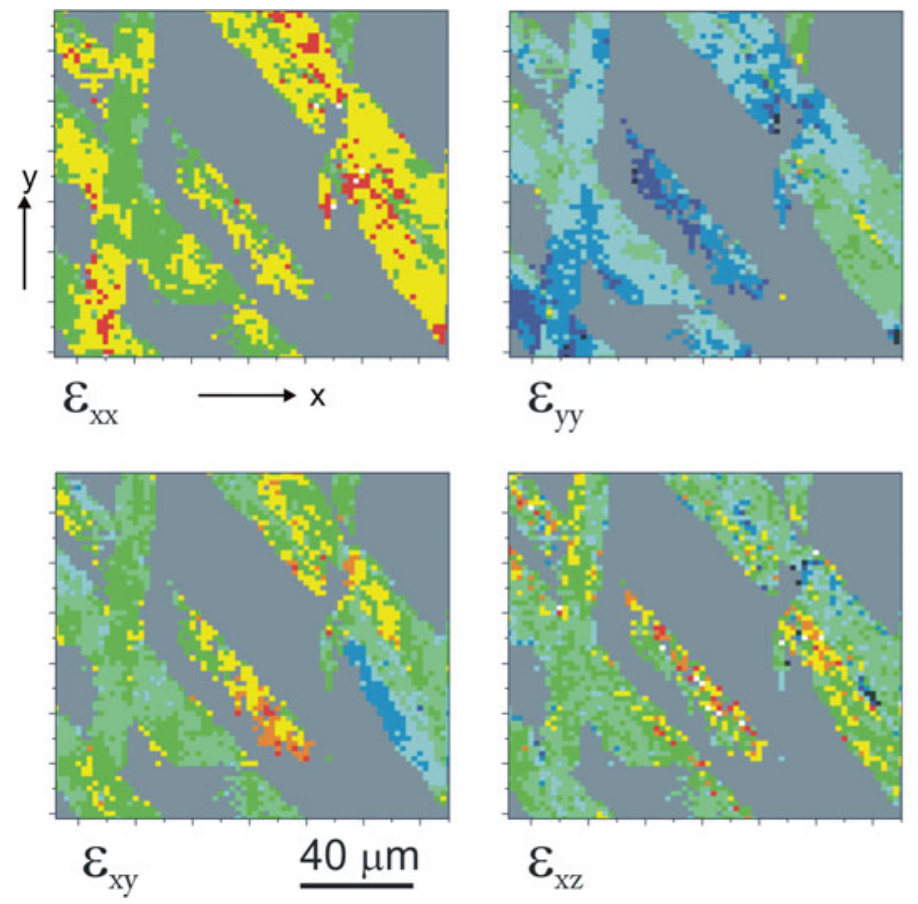

$\varepsilon_{\mathrm{xz}}$

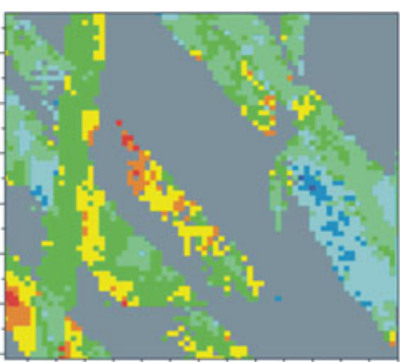

$10^{-3}$
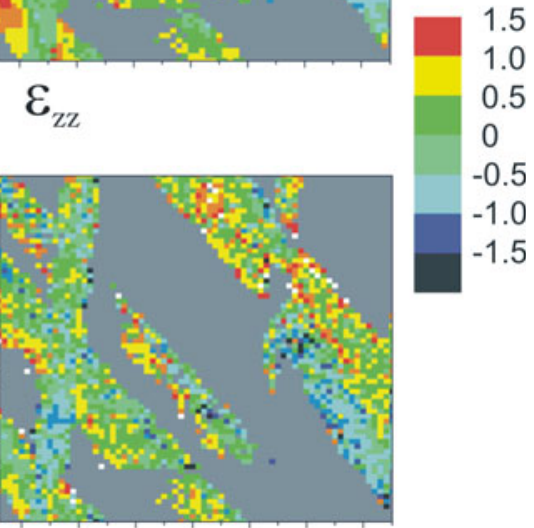

$\varepsilon_{y z}$

Fig. 4 Maps of strain components $\varepsilon_{x x}, \varepsilon_{x y}, \varepsilon_{x z}, \varepsilon_{y y}, \varepsilon_{y z}, \varepsilon_{z z}$ in twin Domains 1, 2, and 3

thus the sample thickness $d e$ is calculated to be $d e=\frac{1}{2}(a d-b c) \cdot \tan 56^{\circ}=47 \mu \mathrm{m}$. Furthermore, the width of the primary twin lamella $c f=\left(\frac{a d-b c}{2}+b c\right) \cdot \sin 56^{\circ}=$ $44 \mu m$.
Maps of the six strain components of Domain 1 are displayed in laboratory coordinates in Fig. 7. From the map, it is seen that the strain magnitude is small (indicated by the green color) in most regions except close to twin 
(a)

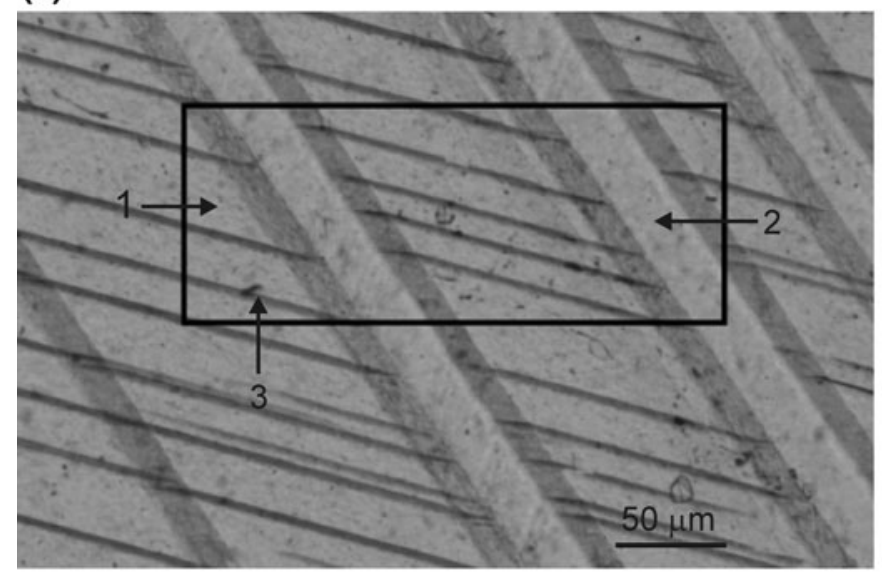

(b)

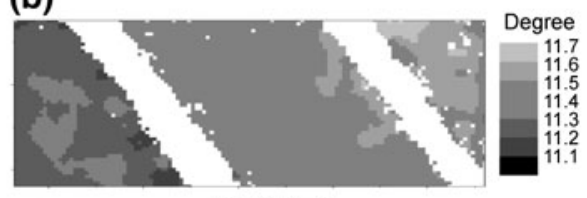

(c)
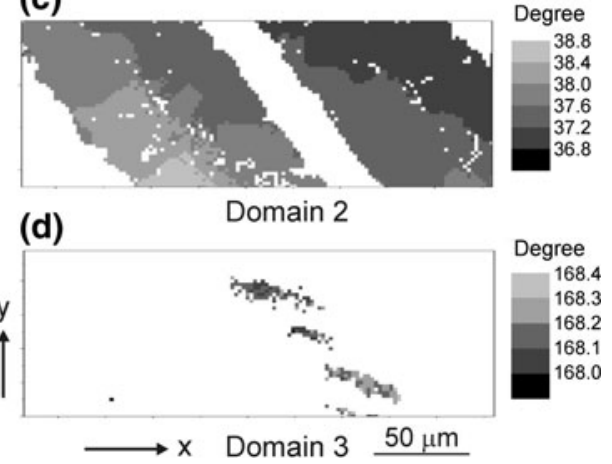

Fig. 5 a Optical micrograph of a calcite grain containing stopping twins. Orientation maps showing the angles $\mathbf{b}$ between crystal $\mathbf{c}$-axis of Domain 1 and laboratory $\mathbf{x}+\mathbf{y}$ direction, $\mathbf{c}$ between crystal $\mathbf{c}$-axis of Domain 2 and laboratory $\mathbf{z}$-axis, $\mathbf{d}$ between crystal $\mathbf{c}$-axis of Domain 3 and laboratory $\mathbf{y}$-axis. The numbered arrows refer to the three domains
Fig. 6 a Stereographic projection showing the $\mathbf{c}$-axis (0001) and $\{1 \overline{1} 08\}$ poles of Domain 1 and corresponding $\{1 \overline{1} 08\}$ poles of Domains 2 and 3. b Schematic of the cross section of the sample perpendicular to a twin plane for Domain 1 and Domain 2 in laboratory coordinates. Letters refer to derivation of domain thickness vf given in the text
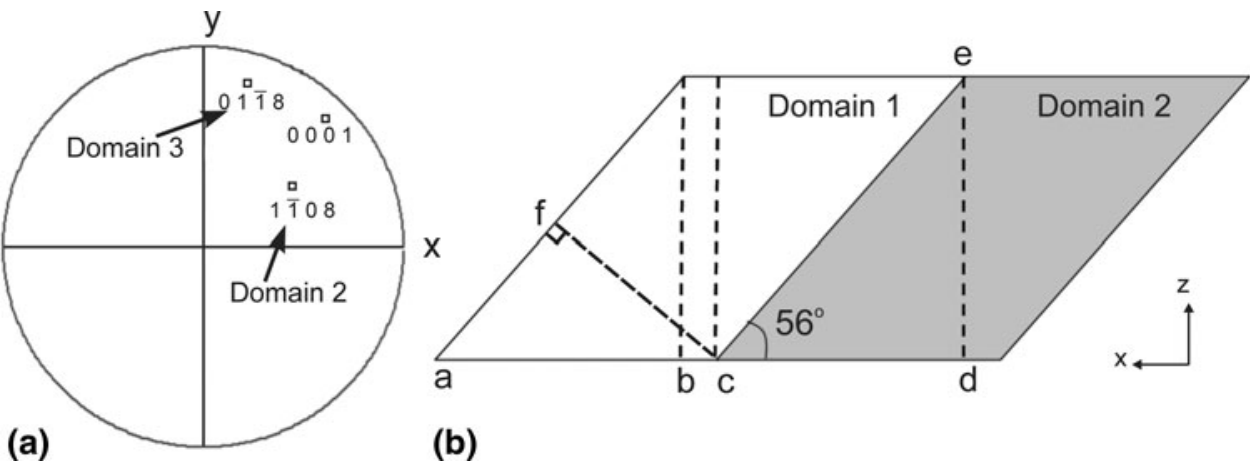

(b)
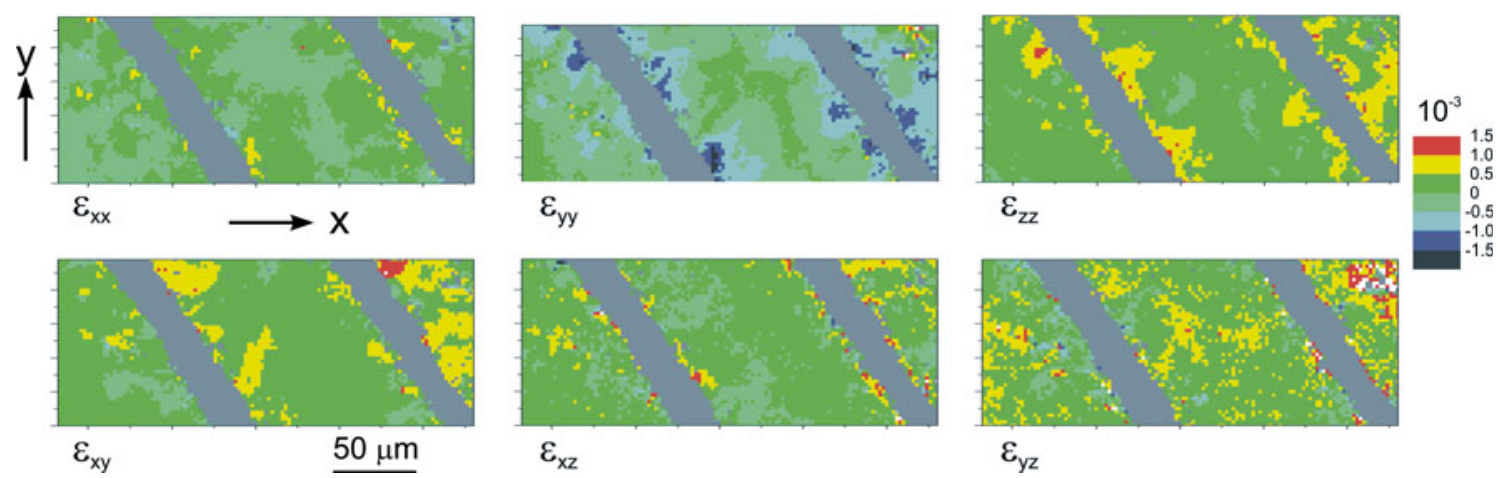

Fig. 7 Maps of strain components $\varepsilon_{x x}, \varepsilon_{x y}, \varepsilon_{x z}, \varepsilon_{y y}, \varepsilon_{y z}$, and $\varepsilon_{z z}$ in Domain 1 in laboratory coordinates $\mathbf{x}, \mathbf{y}$, and $\mathbf{z}$ defined by the sample

lamellae boundaries, where the strain magnitude is approximately $1.5 \times 10^{-3}$.

\section{Discussion}

Figures 3 and 7 show that high strains are observed near to the twin boundaries. The off-diagonal strain components in
Fig. 3 are significantly higher than the diagonal strain components.

In order to further study the strain/stress status of the twin structure, we established a new Cartesian coordinate system, which we call "twin coordinates". We define the $\mathbf{z}$-axis of twin coordinates (denoted as $\mathbf{z}^{\prime}$-axis) perpendicular to twin planes, so that $\mathbf{z}^{\prime}=[\cos \alpha, \cos \beta, \cos \gamma]$, where $\alpha, \beta$, and $\gamma$ are the angles between the twin plane normal 
direction with respect to laboratory $\mathbf{x}-, \mathbf{y}$-, and $\mathbf{z}$-axes, respectively. The $\mathbf{y}$-axis of twinning coordinates ( $\mathbf{y}^{\prime}$-axis) is parallel to the traces of the twin lamellae, i.e., the twin boundaries observed from the $2 \mathrm{D}$ orientation maps, so that $\mathbf{y}^{\prime}=[\cos \theta, \sin \theta, 0]$, where $\theta$ is the angle between the twin lamellae trace direction and laboratory $\mathbf{x}$-axis. The $\mathbf{x}^{\prime}$-axis is perpendicular to both $\mathbf{y}^{\prime}$ - and $\mathbf{z}^{\prime}$-axes, so that $\mathbf{x}^{\prime}=\mathbf{y}^{\prime} \times \mathbf{z}^{\prime}$ (see Figs. 8 and 9 for illustration). The $\mathbf{x}^{\prime} \mathbf{y}^{\prime}$ plane of this twin coordinate system is by definition parallel to the twin planes. However, because three different twin orientations are observed in the crossing twin region, this method is only applied to the stopping twin region (the region described in the "Stopping twins" section), in which the twin coordinate is fixed within the whole scanned area. While the twin plane orientation has been measured and described in laboratory coordinates (Figs. 5, 7), the transformation matrix from laboratory coordinates to twin coordinates is derived as follows:

$\mathbf{R}=\left[\begin{array}{l}\mathbf{x}^{\prime} \\ \mathbf{y}^{\prime} \\ \mathbf{z}^{\prime}\end{array}\right]=\left[\begin{array}{ccc}0.455 & 0.293 & -0.841 \\ -0.542 & 0.840 & 0 \\ 0.707 & 0.456 & 0.541\end{array}\right]$

As described in "Experimental", strain and stress tensors, $\boldsymbol{\varepsilon}_{\mathrm{lab}}$ and $\sigma_{\mathrm{lab}}$, are determined in laboratory coordinates. Thus, strain and stress tensors in twin coordinates, $\boldsymbol{\varepsilon}_{\mathrm{twin}}$ and $\sigma_{\mathrm{twin}}$, can be calculated by Eqs. (4) and (5):

$\varepsilon_{\text {twin }}=\mathbf{R} \cdot \varepsilon_{\text {lab }} \cdot \mathbf{R}^{\mathrm{T}}$

$\sigma_{\text {twin }}=\mathbf{R} \cdot \sigma_{\text {lab }} \cdot \mathbf{R}^{\mathrm{T}}$

Figure 8 shows the stress distributions in Domain 1 in twin coordinates, corresponding to the strain map of Fig. 7 in laboratory coordinates. The stress maps indicate that the normal stresses, $\sigma_{x^{\prime} x^{\prime}}, \sigma_{y^{\prime} y^{\prime}}$, and $\sigma_{z^{\prime} z^{\prime}}$, are higher at the lamellar boundaries than at the center of each lamella, while the shear stresses over the lamellae are homogeneous and low. We attribute this to the release of shear stress during primary twin boundary formation (Cottrell 1965).

Figure 9 displays the stress distribution of Domain 2 in twin coordinates. Stress in Domain 2 is higher than in Domain 1, and similar to Domain 1, the shear stresses in Domain 2 are relatively lower and more homogeneous than normal stresses. Particularly in Domain 1 (Fig. 8), but to some extent also in Domain 2 (Fig. 9), high normal stresses are observed near domain boundaries and the highest concentrations are in clusters. This may be due to interaction with Domain 3 secondary twins which end at these

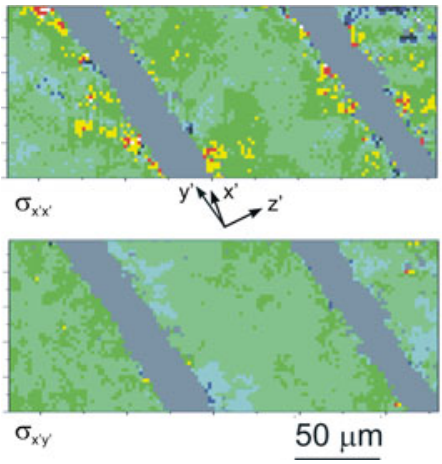

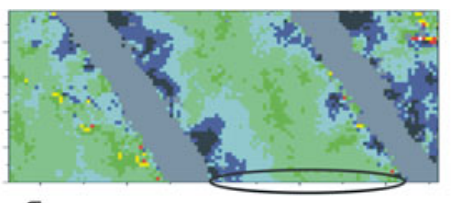

$\sigma_{y^{\prime} y^{\prime}}$

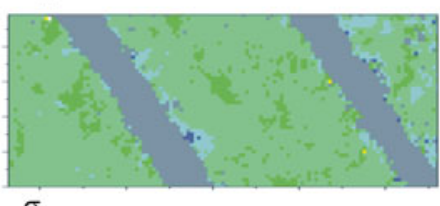

$\sigma_{x^{\prime} z^{\prime}}$

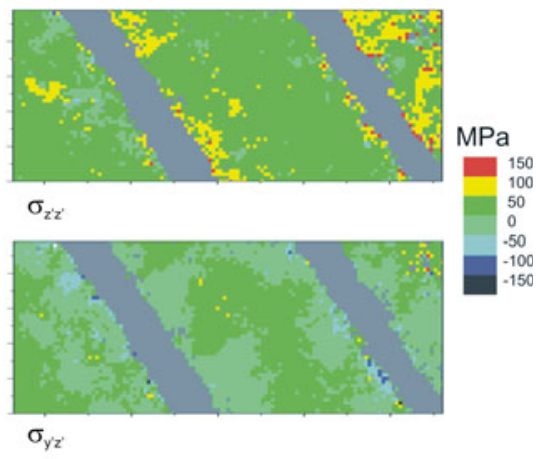

$\sigma_{y^{\prime} z^{\prime}}$

Fig. 8 Maps of stress components $\sigma_{x^{\prime} x^{\prime}}, \sigma_{y^{\prime} y^{\prime}}, \sigma_{z^{\prime} z^{\prime}}, \sigma_{x^{\prime} y^{\prime}}, \sigma_{x^{\prime} z^{\prime}}$, and $\sigma_{y^{\prime} z^{\prime}}$ in Domain 1 , in twin coordinates $\mathbf{x}^{\prime}, \mathbf{y}^{\prime}$, and $\mathbf{z}^{\prime}$. Note that only colored areas refer to Domain 1
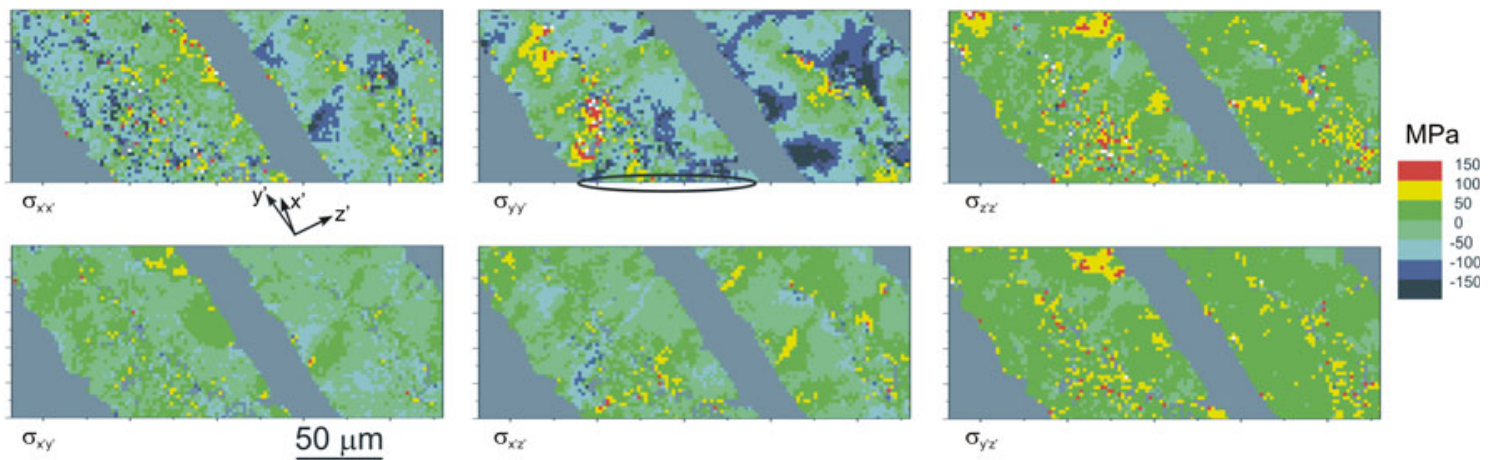

Fig. 9 Maps of stress components $\sigma_{x^{\prime} x^{\prime}}, \sigma_{y^{\prime} y^{\prime}}, \sigma_{z^{\prime} z^{\prime}}, \sigma_{x^{\prime} y^{\prime}}, \sigma_{x^{\prime} z^{\prime}}$, and $\sigma_{y^{\prime} z^{\prime}}$ in Domain 2, in twin coordinates $\mathbf{x}^{\prime}, \mathbf{y}^{\prime}$, and $\mathbf{z}^{\prime}$. Note that only colored areas refer to Domain 2 

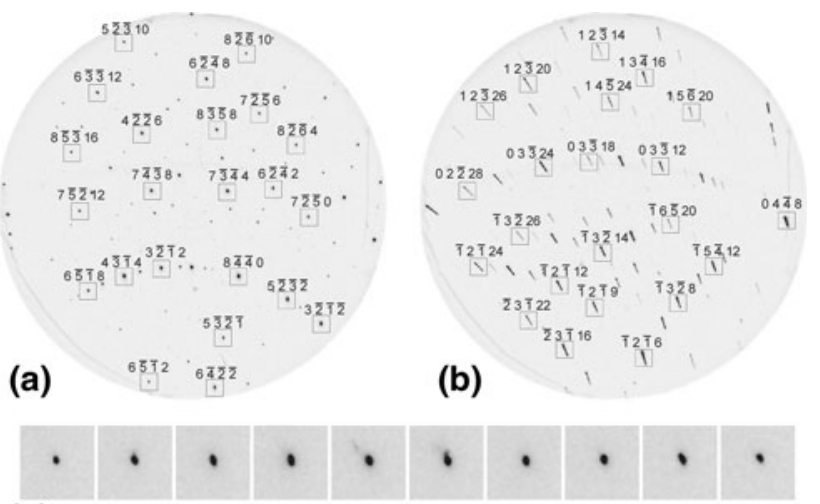

(c)

(d)

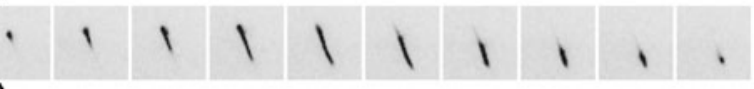

(e)
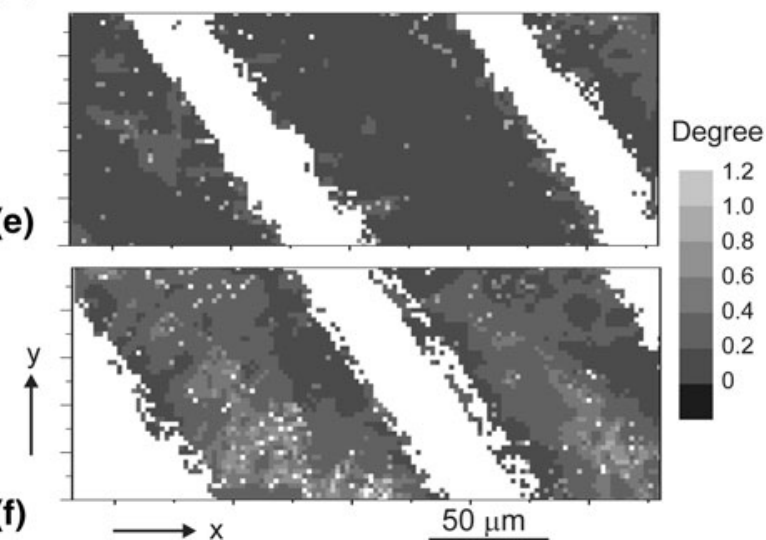

Fig. 10 Typical Laue diffraction patterns from a Domain 1 and b Domain 2. The mosaic image c shows single diffraction peak shape from the circled regions in Fig. 8 (Domain 1) and $\mathbf{d}$ of the circled regions in Fig. 9 (Domain 2) in 10- $\mu$ m steps. e, $\mathbf{f}$ maps of peak shape (long axis in degrees) for Domain 1 (e) and Domain 2 (f)

boundaries. Unfortunately, due to the small size of domain 3 twins, this cannot be quantified. The stress distribution in Domain 2 is less homogeneous than in Domain 1. This coincides with the observation that diffraction peaks from Domain 2 are highly streaked (about $1.5^{\circ}$ ), which is attributed to a high density of geometrically necessary dislocations (GNDs) (Cermelli and Gurtin 2000), so that the strain measurements in
Domain 2 are less accurate than in Domain 1, where diffraction peaks are much sharper. Typical diffraction patterns taken on both domains together with Miller indices of some diffraction peaks are shown in Fig. 10a (Domain 1) and 10b (Domain 2). The shapes of the $(7 \overline{3} \overline{4} 4)$ reflection of Domain 1 and the $(03 \overline{3} 12)$ reflection of Domain 2 from the circled regions in Figs. 8 and 9 are displayed in Fig. 10c, d, respectively, with $10-\mu \mathrm{m}$ step size. Both peaks are close to the center of the CCD detector. The diffraction peaks are highly streaked because the crystal planes are bent around the [0001] axis caused by the GNDs. This was further quantified in the image analysis by fitting each peak with a 2D Lorentzian function and extracting the two main axes in angular values. The average value of the large axis over all indexed reflections in the Laue pattern is mapped in Fig. 10e (Domain 1) and $\mathrm{f}$ (Domain 2). It is evident that the diffraction peaks from Domain 1 are much sharper than from Domain 2. The distortion of the lattice and corresponding streaking of diffraction peaks is caused by dislocations (Barabash et al. 2002, 2004). Simulation results, which are based on the bending of crystal planes induced by the GNDs, indicate that the dislocation line direction is [0001], which is consistent with the assumption that dislocations were imposed in the host domain by twinning, as shown by Barber and Wenk (1979, their Fig. 5a). Therefore, we propose that Domain 2 is the host domain and Domain 1 is the primary twin domain in this case.

Figure 11 shows histograms of equivalent strain (Eq. 1) and equivalent stress (Eq. 2) in the host domain and twin domains in the crossing twin region. In these distributions, the peak equivalent strains (maxima of the distributions) in host domain and twin domains are $0.7 \times 10^{-3}$ and $1.0 \times 10^{-3}$, respectively. The distribution of equivalent strain in twin domains is broader $\left(\mathrm{FWHM}=1.0 \times 10^{-3}\right)$ than in the host domain $\left(\mathrm{FWHM}=0.7 \times 10^{-3}\right)$. The peak equivalent stress in twin domains (150 MPa) is more than twice as high as the peak equivalent stress in host domain (70 MPa), and the stress distribution in twin domains (FWHM $=160 \mathrm{MPa}$ ) is also broader than in the host domain $(\mathrm{FWHM}=110 \mathrm{MPa})$, as shown in Fig. 5b. We attribute this to the fact that the strain is high and non-uniform at the twin boundaries, so that the
Fig. 11 Crossing twin region. a Equivalent strain and b equivalent stress histograms in host and twin domains. Each distribution is normalized such that the maximum is 1.0
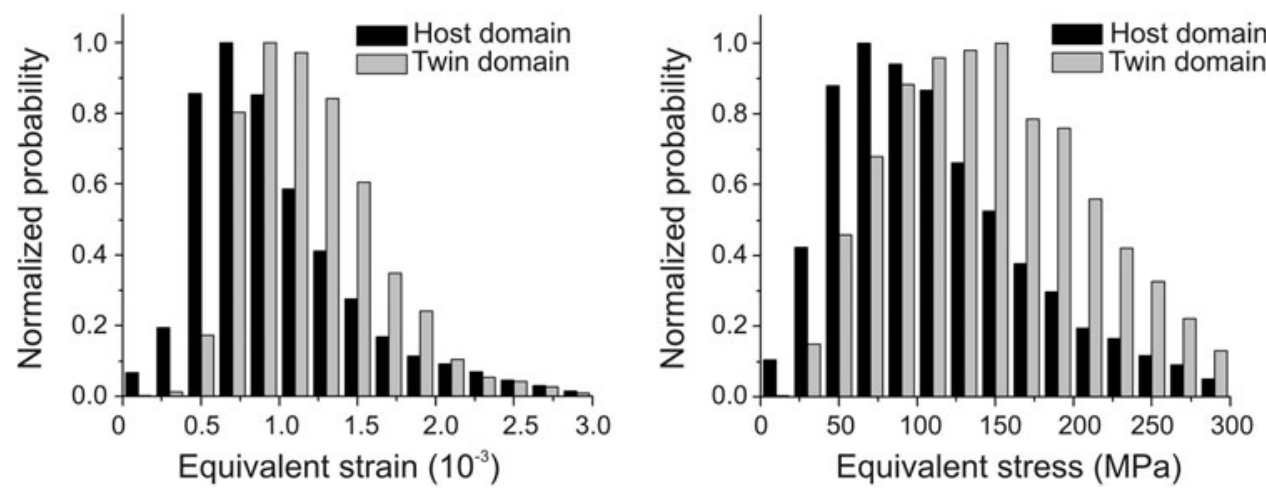
Fig. 12 Stopping twin region a Equivalent strain and b equivalent stress histograms in Domain 1 and Domain 2. Each distribution is normalized such that the maximum is 1.0

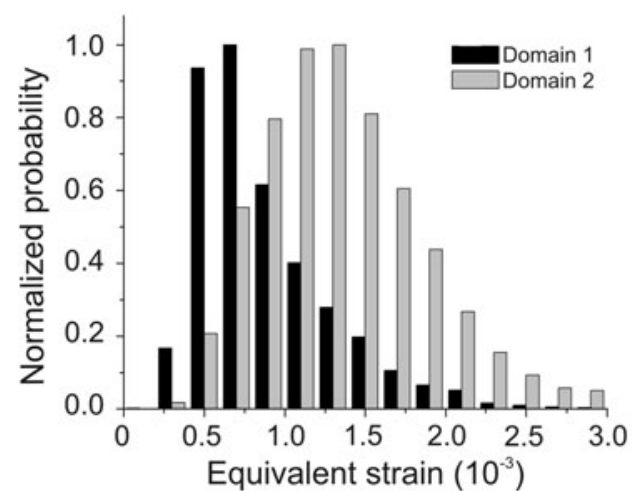

equivalent strain in twin domains is high and scattered, while in the host domain, it is small because it is averaged over the undeformed region.

Equivalent strain (Fig. 12a) and equivalent stress (Fig. 12b) distributions of the primary twin domain (Domain 1) and host domain (Domain 2) in the scanned stopping twin region are shown. The peak equivalent strains of the primary twin domain (Domain 1) and the host domain (Domain 2) are $0.6 \times 10^{-3}$ and $1.2 \times 10^{-3}$, respectively. The distribution of equivalent strain in twin domains is narrower $(\mathrm{FWHM}=$ $\left.0.6 \times 10^{-3}\right)$ than in the host domain $\left(\mathrm{FWHM}=1.2 \times 10^{-3}\right)$. The peak equivalent stress in twin domains (60 MPa) is much lower than the peak equivalent stress in host domain (110 MPa), and the stress distribution in twin domains $(\mathrm{FWHM}=60 \mathrm{MPa})$ is also narrower than in the host domain $(\mathrm{FWHM}=100 \mathrm{MPa})$. Compared to the crossing twin region studied above, the equivalent strain/stress values are smaller in the stopping twin region and the distribution is narrower as well. The stress in the primary twin domain is smaller than in the host domain, though identification of "host" and "twin" is more tentative, as discussed above.

The equivalent stresses reported by other researchers in twinned calcite polycrystals vary widely from smaller than $40 \mathrm{MPa}$ (Lacombe and Laurent, 1996) to greater than $160 \mathrm{MPa}$ (Lacombe and Laurent 1992). The stress measured in the host domains $(\sim 60 \mathrm{MPa})$ in this marble sample is lower than in the quartzite from the Vredefort meteorite impact site in South Africa (90 MPa) but much higher than in quartz from moderately deformed granite (18 MPa) (Chen et al. 2011). Based on observation of residual stresses that are still preserved in this marble, local stresses during twin formation must have exceeded 100-200 MPa during tectonic deformation at metamorphic conditions.

\section{Conclusions}

In this paper, we report microstructural studies of calcite with mechanical twins by using synchrotron X-ray Laue microdiffraction. The sample is from a coarse-grained marble from the Bergell Alps. The aim of this study was to explore the distribution of microstrains that are still preserved. Twin planes $e=\{01 \overline{1} 8\}$ are identified and correspond to the well-known mechanical twins in calcite. The focus was on the strain/stress state associated with mechanical twins. In the crossing twins, it is found that equivalent strain/stress is higher and wider distributed near the twin planes. Interestingly, a detailed study on the stress on stopping twins shows that shear stress is almost completely released on the twin planes close to deformation twin boundaries; however, normal stress is concentrated close to twin planes. Diffraction peaks from the host domain are highly streaked, suggesting high local dislocation densities. It appears that the method of microfocus Laue diffraction has potential to be used as paleopiezometer, on the basis of both residual stress and plastic strain determination.

Acknowledgments We acknowledge support from DOE-BES (DEFG02-05ER15637) and NSF (EAR-0337006) and access to ALS beamline 12.3.2. ALS is supported by the Director, Office of Science, Office of Basic Energy Sciences, Materials Science Division, of the US Department of Energy under Contract No. DE-AC02-05CH11231. The microdiffraction program at the ALS beamline 12.3.2 was made possible by NSF grant \# 0416243. We acknowledge helpful and constructive reviews by Dr. S.J. Covey-Crump and Dr. E. Mariani.

Open Access This article is distributed under the terms of the Creative Commons Attribution Noncommercial License which permits any noncommercial use, distribution, and reproduction in any medium, provided the original author(s) and source are credited.

\section{References}

Amrouch K, Lacombe O, Bellahsen N, Daniel JM, Callot JP (2010) Stress and strain patterns, kinematics and deformation mechanisms in a basement-cored anticline: Sheep Mountain Anticline, Wyoming. Tectonics 29, TC1005

Barabash RI, Ice GE, Larson BS, Yang W (2002) Application of white X-ray microbeams for the analysis of dislocation structures. Rev Sci Instrum 73:1652-1654

Barabash RI, Ice GE, Tamura N, Valek BC, Bravman JC, Spolenak R, Patel JR (2004) Quantitative characterization of electromigrationinduced plastic deformation in $\mathrm{Al}(0.5 \mathrm{wt} \% \mathrm{Cu})$ interconnect. Microelectron Eng 75:24-30 
Barber DJ, Wenk HR (1979) Deformation twinning in calcite, dolomite, and other rhombohedral carbonates. Phys Chem Miner 5:141-165

Burkhard M (1993) Calcite twins, their geometry, appearance and significance as stress-strain markers and indicators of tectonic regime: a review. J Struct Geol 15:351-368

Cermelli P, Gurtin ME (2000) On the characterization of geometrically necessary dislocations in finite plasticity. J Mech Phys Solids 49:1539-1568

Chen CC, Lin CC, Liu LG, Sinogeikin SV, Bass JD (2001) Elasticity of single-crystal calcite and rhodochrosite by Brillouin spectroscopy. Am Mineral 86:1525-1529

Chen K, Kunz M, Tamura N, Wenk HR (2011) Evidence for high stress in quartz from the impact site of Vredefort, South Africa. Eur J Mineral (in press)

Chinn AA, Konig RH (1973) Stress inferred from calcite twin lamellae in relation to regional structure of northwest Arkansas. Geol Soc Am Bull 84:3731-3736

Cottrell AH (1965) Dislocations and plastic flow in crystals. Oxford University Press, London, p 87

De Bresser JHP (1996) Steady state dislocation densities in experimentally deformed calcite materials: Single crystals versus polycrystals. J Geophys Res 101(B10):22189-22201

Evans MA, Groshong RH Jr (1994) A computer program for the calcite strain-gauge technique. J Struct Geol 16:277-281

Ferrill DA (1998) Critical re-evaluation of differential stress estimates from calcite twins in coarse-grained limestone. Tectonophys 285:77-86

González-Casado JM, Gumiel P, Giner-Robles JL, Campos R, Moreno A (2006) Calcite e-twins as markers of recent tectonics: insights from Quaternary karstic deposits from SE Spain. J Struct Geol 28:1084-1092

Graf DL (1961) Crystallographic tables for the rhombohedral carbonates. Am Mineral 46:1283-1316

Jamison WR, Spang JH (1976) Use of calcite twin lamellae to infer differential stress. Geol Soc Am Bull 87:868-872

Kunz M, Tamura N, Chen K, MacDowell AA, Celestre RS, Church MM, Fakra S, Domning EE, Glossinger JM, Kirschman JL, Morrison GY, Plate DW, Smith BV, Warwick T, Yashchuk VV, Padmore HA, Ustundag E (2009a) A dedicated superbend X-ray microdiffraction beamline for materials, geo-, and environmental sciences at the advanced light source. Rev Sci Instrum 80:035108

Kunz M, Chen K, Tamura N, Wenk HR (2009b) Evidence for residual elastic strain in deformed natural quartz. Am Mineral 94:1059-1062

Lacombe O, Laurent P (1992) Determination of principal stress magnitudes using calcite twins and rock mechanics data. Tectonophys 202:83-93
Lacombe O, Laurent P (1996) Determination of deviatoric stress tensors based on inversion of calcite twin data from experimentally deformed monophase samples: preliminary results. Tectonophys 255:189-202

Lacombe O, Angelier J, Laurent P (1992) Determining paleostress orientations from faults and calcite twins: a case study near the Sainte-Victoire Range (southern France). Tectonophys 201:141156

Lacombe O, Malandain J, Vilasi N, Amrouch K, Roure F (2009) From paleostresses to paleoburial in fold-thrust belts: preliminary results from calcite twin analysis in the Outer Albanides. Tectonophys 475:128-141

Larsson AK, Christy AG (2008) On twinning and microstructures in calcite and dolomite. Am Mineral 93:103-113

Laurent Ph, Bernard Ph, Vasseur G, Etchecopar A (1981) Stress tensor determination from the study of e twins in calcite: a linear programming method. Tectonophys 78:651-660

Liu AF (2005) Mechanics and mechanism of fracture. ASM International, Schaumburg

Liu W, Ice GE, Tischler JZ, Khounsary A, Liu C, Assoufid L, Macrander AT (2005) Short focal length Kirkpatrick-Baez mirrors for a hard X-ray nanoprobe. Rev Sci Instrum 76:113701

Mügge O (1883) Beiträge zur Kenntnis der Strukturflaechen des Kalkspathes, Neues Jb Mineral 1: 32-54, 81-85

Noyan IC, Cohen JB (1987) Residual stress-measurement by diffraction and interpretation. Springer, Heidelberg

Pfaff F (1859) Versuche über den Einfluß des Drucks auf die optischen Eigenschaften doppeltbrechender Krystalle. Ann Phys 107:333-338

Rowe KJ, Rutter EH (1990) Palaeostress estimation using calcite twinning: experimental calibration and application to nature. J Struct Geol 12:1-17

Spang JH (1972) Numerical method for dynamic analysis of calcite twin lamellae. Geol Soc Am Bull 83:467-472

Spang JH (1974) Numerical dynamic analysis of calcite twin lamellae in the Greenport Center Syncline. Am J Sci 274:1044-1058

Spang JH, Van Der Lee J (1975) Numerical dynamic analysis of quartz deformation lamellae and calcite and dolomite twin lamellae. Geol Soc Am Bull 86:1266-1272

Tamura N, MacDowell AA, Spolenak R, Valek BC, Bravman JC, Brown WL, Celestre RS, Padmore HA, Batterman BV, Patel JR (2003) Scanning X-ray microdiffraction with submicrometer white beam for strain/stress and orientation mapping in thin films. J. Synchrotron Rad 10:137-143

Tamura N, Kunz M, Chen K, Celestre RS, MacDowell AA, Warwick $\mathrm{T}$ (2009) A superbend X-ray microdiffraction beamline at the advanced light source. Mat Sci Eng A-Struct 524:28-32 